\title{
Cutaneous mechanisms of isometric ankle force control
}

\author{
Julia T. Choi $\cdot$ Jesper Lundbye-Jensen • \\ Christian Leukel · Jens Bo Nielsen
}

\begin{abstract}
The sense of force is critical in the control of movement and posture. Multiple factors influence our perception of exerted force, including inputs from cutaneous afferents, muscle afferents and central commands. Here, we studied the influence of cutaneous feedback on the control of ankle force output. We used repetitive electrical stimulation of the superficial peroneal (foot dorsum) and medial plantar nerves (foot sole) to disrupt cutaneous afferent input in 8 healthy subjects. We measured the effects of repetitive nerve stimulation on (1) tactile thresholds, (2) performance in an ankle force-matching and (3) an ankle position-matching task. Additional forcematching experiments were done to compare the effects of transient versus continuous stimulation in 6 subjects and to determine the effects of foot anesthesia using lidocaine in another 6 subjects. The results showed that stimulation decreased cutaneous sensory function as evidenced by increased touch threshold. Absolute dorsiflexion force
\end{abstract}

J. T. Choi · J. Lundbye-Jensen · J. B. Nielsen $(\triangle)$

Department of Nutrition, Exercise and Sports,

University of Copenhagen, Panum Institute 33.3,

Blegdamsvej 3, 2200 Copenhagen N,

Denmark

e-mail: jbnielsen@sund.ku.dk

J. T. Choi $\cdot$ J. Lundbye-Jensen $\cdot$ J. B. Nielsen

Department of Neuroscience and Pharmacology, University of

Copenhagen, Copenhagen, Denmark

C. Leukel

Department of Sport Science, University of Freiburg, Freiburg, Germany

\section{Leukel}

Department of Medicine, Movement and Sport Science, University of Fribourg, Fribourg, Switzerland error increased without visual feedback during peroneal nerve stimulation. This was not a general effect of stimulation because force error did not increase during plantar nerve stimulation. The effects of transient stimulation on force error were greater when compared to continuous stimulation and lidocaine injection. Position-matching performance was unaffected by peroneal nerve or plantar nerve stimulation. Our results show that cutaneous feedback plays a role in the control of force output at the ankle joint. Understanding how the nervous system normally uses cutaneous feedback in motor control will help us identify which functional aspects are impaired in aging and neurological diseases.

Keywords Cutaneous feedback · Force .

Ankle dorsiflexion $\cdot$ Human

\section{Introduction}

The sense of force is critical in our interactions with the physical environment. For example, we use an estimate of the amount of force applied to move an object (e.g., pushing a shopping cart) to determine whether it is heavy or light. Force perception depends on the interaction between peripheral feedback and central motor commands; perceived force magnitude can be biased by removing cutaneous feedback via anesthesia (Gandevia and Mccloskey 1977a, b; Marsden et al. 1979), during fatigue (Jones and Hunter 1983) or after central lesions (Maschke et al. 2006; Simon et al. 2009).

The control of finger force output can be achieved to some extent in the absence of peripheral feedback, as demonstrated in a man with peripheral neuropathy (Rothwell et al. 1982). Patients with sensory neuropathy instead rely 
on visual feedback to maintain accuracy (Rothwell et al. 1982; Sanes et al. 1984). In order to achieve fine force resolution in finger control, cutaneous feedback must be properly integrated with central motor command (Henningsen et al. 1995, 1997). Besides the importance of cutaneous feedback in fine finger force control, less is known about the relevance of cutaneous afferents for controlling force at the ankle.

In the lower limbs, the effects of deafferentation via anesthesia suggest that the nervous system has the means to adjust the motor command to produce different levels of muscle activity without peripheral information, but cannot maintain the constant output over time (Gandevia et al. 1993). We therefore hypothesized that cutaneous feedback is normally used in ankle force control, and we predicted that force errors would increase with disruption of cutaneous feedback.

To investigate the importance of cutaneous feedback for ankle force control, we disrupted afferent signals from the foot using repetitive electrical nerve stimulation. The advantage of using electrical nerve stimulation, rather than anesthesia as in previous experiments, is that it could be turned on and off, enabling a quick switch between normal and altered cutaneous feedback conditions. Before testing the effects of electrical stimulation on ankle force control, we determined the effects of the stimulation on cutaneous and proprioceptive function by measuring touch and vibration thresholds. Repetitive stimulation of the peroneal nerve and plantar nerve increased touch thresholds on the foot dorsum and foot sole, respectively. Second, we found that absolute ankle dorsiflexion force errors increased during peroneal nerve stimulation without vision. The effect of stimulation was not observed in ankle position matching. The results suggest that ankle force control, like finger force control, depends on the integration of cutaneous afferents feedback.

\section{Methods}

\section{Subjects}

The study was approved by the local ethics committee of the Copenhagen region (Protokol nr. H-A-2008-029) and conformed to the Declaration of Helsinki. Twenty healthy subjects ( 7 females, 13 males; mean age \pm SD $27 \pm 5$ year) were recruited from the University of Copenhagen campus. All subjects gave written informed consent prior to participation.

Participants were divided into three groups. In the first group of subjects $(n=8)$, we tested tactile thresholds, force matching and position matching with transient nerve stimulation. In the second group of subjects $(n=6)$, we tested force matching with transient stimulation versus continuous nerve stimulation. In a third group of subjects $(n=6)$, force matching was tested before and after lidocaine injection.

\section{Repetitive nerve stimulation}

Repetitive nerve stimulation was administered with a constant current stimulator (DS7A, Digitimer, UK) that delivered $1 \mathrm{~ms}$ pulses at $80 \mathrm{~Hz}$ through two surface electrodes (2.5 cm PALS Platinum, Axelgaard, Denmark). Stimulation intensity was set at the radiating threshold for each subject. Radiating threshold for each subject was determined by increasing stimulation intensity until the subject perceived a tingling sensation on the skin that radiated to the great toe distal to the stimulation site. Radiating thresholds were determined separately for the foot dorsum and foot sole (peroneal nerve and plantar nerve stimulation, respectively). Two electrodes were placed in the front of the ankle approximately $3 \mathrm{~cm}$ apart to stimulate the medial cutaneous branch of the superficial peroneal nerve. To stimulate the medial plantar nerve (i.e., a branch of the tibial nerve), one electrode was placed below the medial malleolus and another was placed behind the medial malleolus. Stimulation was applied on the right foot.

\section{Touch and vibration thresholds}

Sensory thresholds in two different modalities of touch, pressure and vibration, were measured using common clinical methods. Touch thresholds were measured using Von Frey monofilaments (20 piece Touch Test ${ }^{\mathrm{TM}}$ Sensory Evaluator, North Coast Medical). Areas tested included the foot dorsum, the plantar side of the great toe and heel on the right side. The monofilament was pressed on the skin surface for $1 \mathrm{~s}$ and then removed. Subjects were instructed to say "yes" when the pressure was felt. For each site, increasingly larger monofilaments were used until a single positive response was recorded. For monofilaments size 1.65 through 4.08, corresponding to $0.008-1 \mathrm{~g}$ in force, the touch was applied up to three times. For monofilaments size 4.17-6.65, corresponding to $1.4-300 \mathrm{~g}$ in force, the touch was applied only once. The force from the smallest monofilament to elicit a positive response was the touch threshold. All sites were first tested without stimulation. The foot dorsum was re-tested with peroneal nerve stimulation; the great toe and heel was re-tested with plantar nerve stimulation.

Vibration sensation on the right great toe was determined using Vibratron II device (Physitemp Instruments, New Jersey, USA), which consists of two separate vibrating rods $(100 \mathrm{~Hz})$. Only one rod vibrated at a time. A controller was used to switch which rod will vibrate and to 
adjust the vibration amplitude (0-200 micron). On each trial, the subject pressed the right great toe against each rod in sequence for about $1 \mathrm{~s}$, and then determined which of the two rods was vibrating. When switching between rods, the subject was instructed to be consistent in the location of the touch and the approximate force applied to the great toe. Vibration threshold was measured using a two-alternative forced-choice procedure (Arezzo et al. 1985). The vibration amplitude was decreased by about $10 \%$ from trial to trial, until the subjects made the first incorrect choice. After the first error, each amplitude setting was repeated for a total of 3 trials. The next amplitude was decreased by $10 \%$ if the subject was correct in two out of three trials, and increased by $10 \%$ otherwise. The test was completed when the subject made a total of 5 errors. We noted the vibration amplitudes where the 5 errors were made and where the 5 lowest correct responses were made; the vibration threshold was then calculated as the average of these 10 values, excluding the highest and lowest values (Arezzo et al. 1985). The reproducibility of this method for measuring vibration threshold has a coefficient of variation between 17 and $20 \%$ (Nasseri et al. 1998). The test was performed first without stimulation and then repeated with stimulation of the right peroneal and plantar nerves together.

Force matching

Subjects were seated in an upright position with the right foot attached to a pedal instrumented with a strain gauge force transducer. The knee and ankle angles were approximately $110^{\circ}$ and $120^{\circ}$, respectively. To determine maximal voluntary contraction (MVC), subjects were instructed to exert maximal isometric ankle dorsiflexion force and hold it for approximately $2 \mathrm{~s}$. The peak torque obtained across 3 trials was taken as the MVC.

During the force-matching task, subjects were instructed to maintain an isometric ankle dorsiflexion force equivalent to $10 \%$ maximal voluntary contraction. Subjects watched a monitor displaying the target force represented as a horizontal cursor, and a trace of the actual force applied to the pedal. Each trial consisted of 3 epochs: 0-5 s (eyes opened, EO), 5-10 s (eyes closed, EC1) and 10-15 s (eyes closed, EC2). Electrical stimulation was applied at the same time visual feedback was removed (at $5 \mathrm{~s}$ ). Subjects completed a total of 15 trials: 5 trials in each of the 3 stimulation conditions (no stimulation, right peroneal nerve and right plantar nerve) randomly.

\section{Effects of transient versus continuous stimulation}

We hypothesized that the nervous system may compensate for disrupted cutaneous feedback by using other sources of information (e.g., visual feedback, muscle afferents, central motor commands) to recalibrate the force output. To test this, we compared the effects of disrupting cutaneous feedback via transient versus continuous peroneal nerve stimulation in subjects $(n=6)$ who did not participate in the first set of force-matching experiments. In the former condition, stimulation was turned at the same time visual feedback was removed, so subjects never received feedback about their performance in the altered cutaneous feedback state. In the latter condition, subjects received feedback about their performance in the altered cutaneous feedback state before visual feedback was removed, and therefore had time to recalibrate the force output.

As before, the force-matching trial consisted of 3 epochs: EO (0-5 s), EC1 (5-10 s) and EC2 (10-15 s). Each subject completed 4 blocks of 5 trials (a total of 20 trials) in the same order: no stimulation was applied (e.g., removing visual feedback only) in block 1; peroneal nerve stimulation was applied starting at 0 s (e.g., disrupting cutaneous feedback before removing visual feedback) in block 2; no simulation was applied in block 3; peroneal nerve stimulation was applied starting at $5 \mathrm{~s}$ (e.g., disrupting cutaneous feedback and visual feedback at the same time) in block 4 . The effects of continuous versus transient repetitive electrical nerve stimulation were determined by comparing blocks 2 and 4. The purpose of block 3 was to washout possible aftereffects from stimulation before re-test.

\section{Effect of nerve block with lidocaine}

A lidocaine experiment was done separately to determine the effects of blocking cutaneous afferents using anesthesia, which does not involve the possibility of activating central mechanisms via peripheral nerve stimulation. The force-matching task was tested in 6 naive subjects $(n=6)$ before and after anesthetizing of the foot. Lidocaine $(25 \mathrm{mg} / \mathrm{ml})$ was injected around the ankle joint targeting the deep peroneal nerve, branches of the superficial peroneal nerve and the tibial nerve. Areas on the foot dorsum and foot sole were tested for sensation of light touch (i.e., short finger strokes). Complete anesthesia of the whole foot was obtained in two subjects. In the remaining subjects, lack of sensation was obtained in approximately $80-90 \%$ of the plantar and dorsal sides of the foot, but complete anesthesia of the toes and the heel was not obtained. Another evaluation of light touch was done at the end to ensure that the anesthesia was effective throughout the experiment. The force-matching trial consisted of 3 epochs: EO (0-5 s), EC1 (5-10 s) and EC2 (10-15 s). Each subject completed 3 blocks of 5 trials (total of 15 trials): pre-lidocaine injection, post-lidocaine injection and post-lidocaine injection with peroneal nerve stimulation (starting at $5 \mathrm{~s}$ ). 
Position matching

We tested whether cutaneous stimulation affected ankle position sense. Subjects were seated in an upright position with both feet placed in separate pedals allowing independent movement of the ankle joints. Ankle angles were measured using electrogoniometer bilaterally and recorded at $2 \mathrm{kHz}$. The rotational axis of each pedal was aligned to the rotational axis of the ankle. The knee angle was fixed at approximately $110^{\circ}$. The ankle angle was approximately $90^{\circ}$ at the starting position. The experimenter rotated the subject's left ankle (dorsiflexion) from the starting position to a random position within normal range of motion. The rotation speed varied across trials in order to decouple the test position and the duration of rotation. While being blindfolded, the subject was asked to move their right ankle to match the left ankle angle position and hold it for approximately $2 \mathrm{~s}$. Then, both ankles were lowered to the starting position separately. There were 60 trials in total: 20 random positions in each of the 3 stimulation conditions (no stimulation, right peroneal nerve and right plantar nerve). The stimulation was applied at the beginning of each trial and lasted for $5 \mathrm{~s}$.

\section{Data analysis}

The Wilcoxon signed-rank test was used to compare touch and vibration thresholds between the stimulation $\mathrm{ON}$ and OFF conditions. An alpha-level of 0.05 was used as the statistical significant criterion for all comparisons.

In the force-matching task, error was calculated as the mean absolute difference between actual and target force during three epochs: 3-5 s (eyes opened, EO), 5-10 s (eyes closed, EC1) and 10-15 s (eyes closed, EC2). A two-way repeated measures ANOVA with the factors epoch (EO, $\mathrm{EC} 1, \mathrm{EC} 2$ ) and stimulation (no stimulation, peroneal nerve, plantar nerve) was used to compare force error. When the ANOVA yields a significant effect, post hoc analysis comparing the means against the control value (EO, no stimulation) was performed with a Dunnett's test.

In the angle-matching task, error was calculated as the absolute angular difference between the actual (left) ankle and the reported (right) ankle. The mean position was taken from the hold period, determined using the velocity of the right ankle joint. Sometimes, subjects made one or two small adjustments after the first stop. In such case, the final position was taken from the last hold period. Subjects always reached the final position before the end of the 5-s trial. A one-way repeated measures ANOVA with the factor stimulation (no stimulation, peroneal nerve, plantar nerve) was used to compare position error.

\section{Results}

\section{Touch threshold}

Touch sensation was impaired with repetitive stimulation of the superficial peroneal and medial plantar nerves (Fig. 1a). Peroneal nerve stimulation increased touch threshold on the foot dorsum $(P=0.02)$. Plantar nerve stimulation increased touch threshold on the great toe $(P=0.02)$, but not the heel $(P=0.8)$. This corresponded to an increase in monofilament size from 3.61 to 4.31 for the foot dorsum and from 3.61 to 4.08 for the great toe. This demonstrated that peroneal nerve stimulation and plantar nerve stimulation impaired cutaneous sensation in the foot dorsum and foot sole, respectively.
Fig. 1 Touch and vibration thresholds. a Group-average touch thresholds are plotted for stimulation $\mathrm{ON}$ and OFF conditions. Areas of the skin tested are illustrated in the inset. $\mathbf{b}$ Group-average vibration thresholds are plotted for stimulation $\mathrm{ON}$ and OFF conditions. Horizontal dotted line represents 2 $\mathrm{SD}$ above the mean of normal values reported in the literature (Arezzo et al. 1985). Colored circles represent individual subjects. Error bars represent group standard errors (SE). Asterisk indicates $P<0.05$; $n s$ not significant (color figure online)
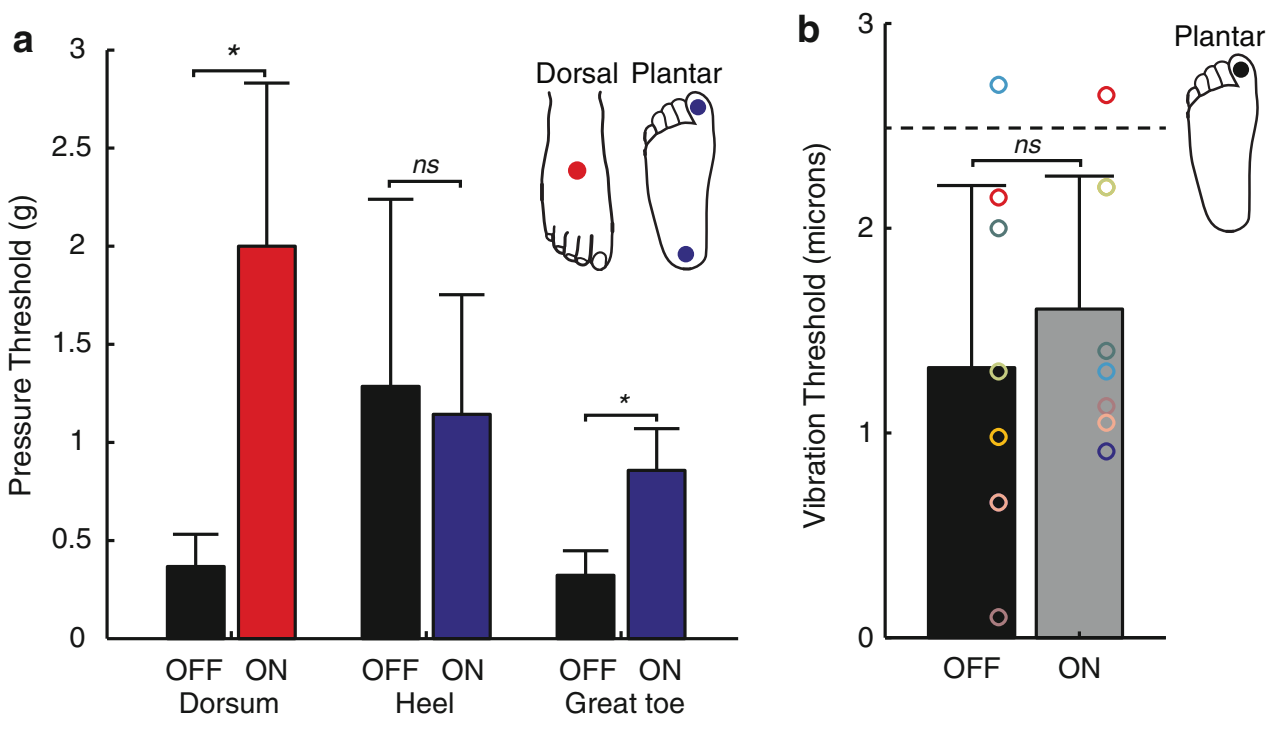
Fig. 2 Force matching. a Group-average absolute force error across time epochs (EO, EC-1, EC-2) with no stimulation (black), peroneal nerve stimulation (gray) and plantar nerve stimulation (white). Bottom, shaded area represents period without visual feedback. Dotted line begins and ends at the time of stimulation onset and offset, respectively. b Group-average absolute force error with no stimulation (black), continuous stimulation (light gray) and transient stimulation (dark gray) of the peroneal nerve stimulation. c Group-average absolute force error pre-lidocaine (black) and post-lidocaine with no stimulation (light gray) and peroneal nerve stimulation (dark gray). Colored dots represent individual subjects. Error bars represent group SE (color figure online)

\section{Vibration threshold}

Repetitive electrical nerve stimulation did not have a robust effect on vibration sensation. The group-average vibration threshold increased approximately $20 \%$, but the effect was not statistically significant (Fig. $1 \mathrm{~b}, P=0.4$ ). Group-average vibration thresholds measured with and without stimulation were both within the range of normal values reported in the literature (Arezzo et al. 1985). Therefore, repetitive electrical nerve stimulation did not significantly impair vibration sensation.

\section{Force matching}

Subjects could normally maintain a constant force in the absence of visual feedback. However, the absolute dorsiflexion force error increased when cutaneous feedback was disrupted during peroneal nerve stimulation. Group data showed a significant difference in absolute force error across stimulation conditions (Fig. 2a). Repeated measures ANOVA showed a significant effect of epoch $(F[2,10]=25, P<0.001)$, stimulation $(F[2,10]=4.4$, $P=0.04)$ and interaction between epoch and stimulation $(F[4,20]=3.7, P=0.02)$ on the force errors. Post hoc analysis showed that force errors increased during the eyesclosed epochs, EC1 $(P<0.05)$ and EC2 $(P<0.001)$, with peroneal nerve stimulation. There was no significant effect of plantar nerve stimulation, suggesting that it was not a general effect of repetitive nerve stimulation. The results suggest that disrupting cutaneous input from the foot dorsum via peroneal nerve stimulation, but not by plantar nerve stimulation, impaired the ability to estimate dorsiflexion force output.

The mean force was also analyzed in order to determine whether there was consistent over-estimate or under-estimate of force across trials, which would indicate that the stimulation biased cutaneous feedback, rather than disrupting it. Repeated measures ANOVA showed no significant effect of epoch $(F[2,10]=0.06, P=0.9)$ or stimulation $(F[2,10]=2.4, P=0.2)$ on the mean ankle force produced. This suggests that the stimulation did not amplify the
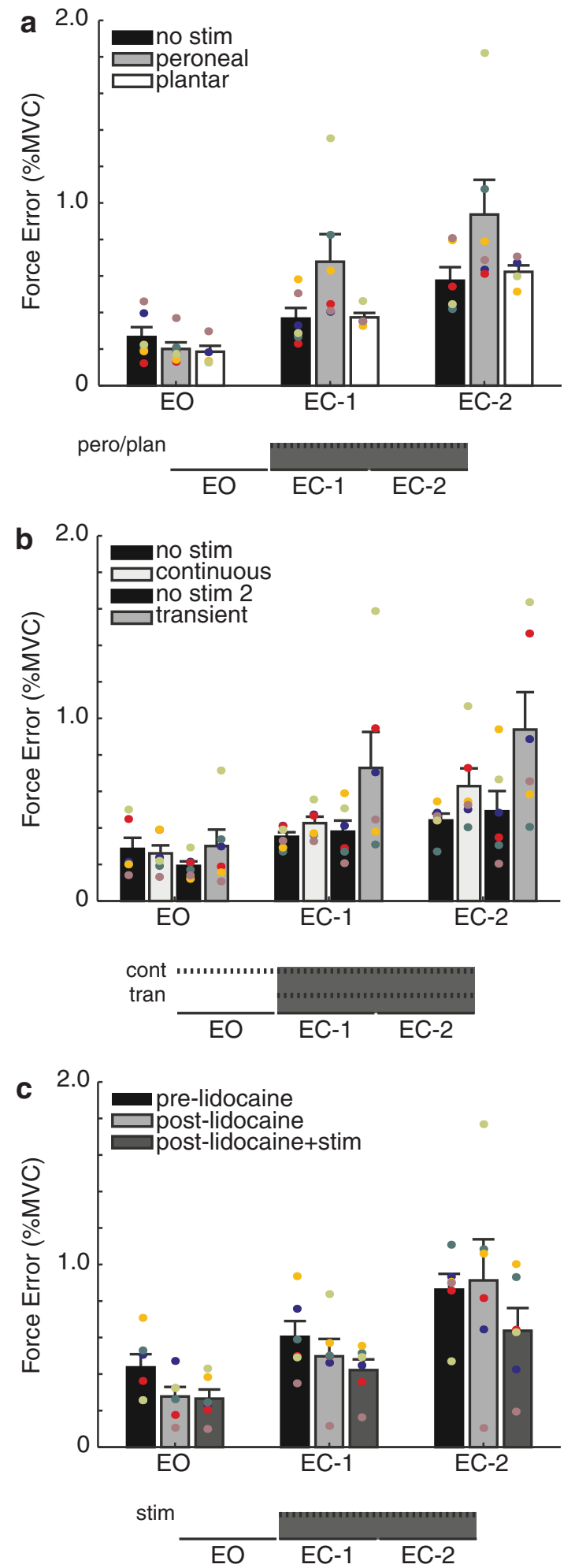

cutaneous afferent signals, which would have caused a consistent over-estimate of force (and thus a decrease in force output when the peroneal nerve was stimulated during the matching task). 
Transient versus continuous stimulation

In a separate group of subjects, we compared the effects of transient stimulation versus continuous peroneal nerve stimulation. The purpose was to test whether subjects compensate for disrupted cutaneous feedback by using visual feedback.

Group data showed a significant difference in absolute force error across stimulation conditions (Fig. 2b). A two-way repeated measures ANOVA showed a significant effect of epoch $(F[2,10]=24, P<0.001)$ and stimulation $(F[3,15]=4.4, P=0.02)$ on the force errors. The interaction effect was non-significant $(F[6,30]=2.3, P=0.06)$.

The results showed that sudden, but not continuous, disruption of cutaneous feedback had a significant effect on force performance. This suggests that the nervous system has the capability of using efferent signals and afferent feedback other than cutaneous afferents to control ankle force output, when sufficient information is provided for the recalibration (see "Discussion").

\section{Lidocaine experiment}

The force-matching task was tested in another group of subjects before and after anesthesia of the foot. The purpose of this experiment was to verify our findings using another method that does not involve the possibility of activating central mechanisms via peripheral nerve stimulation.

Group data showed no significant difference in absolute force error across the three conditions: pre-lidocaine, post-lidocaine, post-lidocaine with stimulation (Fig. 2c). Repeated measures ANOVA showed a significant effect of epoch $(F[2,10]=36, P<0.001)$. The effects of condition $(F[2,10]=2.0, P=0.2)$ and interaction between epoch and condition $(F[4,20]=0.8, P=0.5)$ were not significant.

Since the testing blocks were not randomized, change in performance from the first to the second block is the sum of the effects of lidocaine and practice time. If subjects learned to perform better over time, the effect of practice time may mask the effect of lidocaine. While that is possible, we showed above that errors increased in Block 4 when transition stimulation was applied. This suggests that, if present, the effects of lidocaine were smaller compared to transition stimulation. Lidocaine, like continuous stimulation, did not significantly decrease force accuracy since subjects had time to recalibrate the force output using visual feedback.

The results verified that peroneal nerve stimulation had no effect on force errors after blocking cutaneous feedback with lidocaine injection, suggesting that the effect of nerve stimulation on force output could be attributed to disruption of cutaneous afferents.

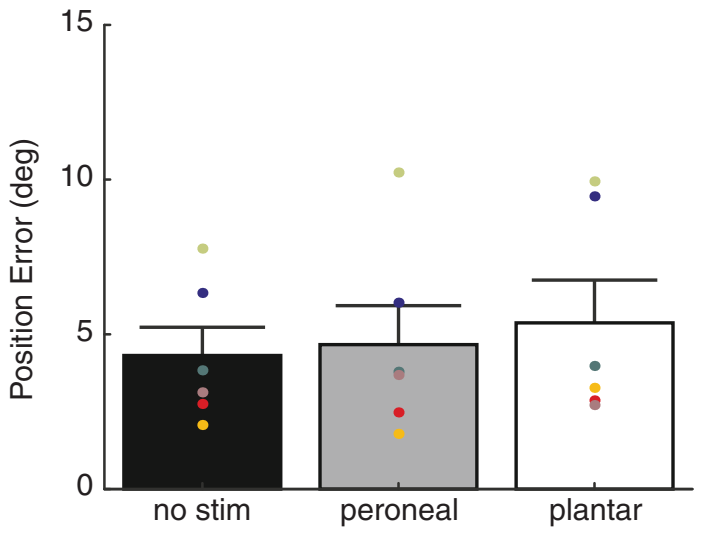

Fig. 3 Position matching. Group-average mean ankle error with no stimulation (black), peroneal nerve stimulation (gray) and plantar nerve stimulation (white). Colored dots represent individual subjects. Error bars represent group SE (color figure online)

\section{Position matching}

Repetitive electrical nerve stimulation did not affect performance in the position-matching task. Group-average data showed that position error is about $4^{\circ}$ (Fig. 3). Repeated measures ANOVA found no significant effect of stimulation $(F[2,10]=1.9, P=0.2)$ on absolute position error. This suggests that repetitive electrical nerve stimulation impaired force estimation, but not position estimation.

\section{Discussion}

We have demonstrated that repetitive electrical nerve stimulation could be used to disrupt cutaneous sensory function. Stimulation at $80 \mathrm{~Hz}$ increased touch threshold on the foot dorsum by about fourfold. When cutaneous feedback was disrupted using repetitive electrical nerve stimulation, ankle force errors increased in the force-matching task. This suggests that subjects normally relied on cutaneous feedback to control force output. With continuous stimulation, but not transient stimulation, subjects were able to compensate for the disrupted cutaneous information with the available visual feedback. Finally, when peroneal nerve stimulation was applied after lidocaine injection, there was no additional effect on force performance. This suggests that the effect of nerve stimulation on force output was attributed to decreased cutaneous sensory function.

The extent of sensory disruption caused by repetitive electrical nerve stimulation was determined by measuring touch and vibration perception thresholds. During stimulation, subjects perceived a "tingling" sensation that resembles the "flutter vibration" and "sustained pressure" sensations reported during microstimulation of rapidly adapting and slowly adaptation cutaneous afferents (Ochoa and 
Torebjörk 1983; Vallbo et al. 1984; Macefield et al. 1990). Our results showed that touch thresholds increased during stimulation, indicating that it was more difficult to perceive mechanically applied pressure on the skin in the presence of the electrically induced sensation. Stimulation did not have a robust effect on vibration thresholds. Vibration sense may be affected to a lesser extent compared to pressure sense because of differences in firing properties and location of the mechanoreceptors and central processing of the sensory afferents. It is also possible that higher stimulation frequencies $(>80 \mathrm{~Hz})$ may be required to disrupt vibration $(100 \mathrm{~Hz})$ thresholds.

\section{Force control}

Previous studies have shown that the perception of weight can be biased after removing cutaneous feedback by anesthesia (Gandevia and Mccloskey 1977a, b; Marsden et al. 1979). The changes in weight perception reflected the corresponding changes in the agonist and antagonist muscle activity after anesthesia (Marsden et al. 1979), rather than the relative contributions of stretch reflex and central motor commands to motorneurons (Gandevia and Mccloskey $1977 \mathrm{a}, \mathrm{b})$. Thus, the size of central commands cannot entirely account for the perception of force, indicating that afferent feedback mechanisms must also play a role in assessing the force output.

The role of afferent feedback in force control has been studied by examining the ability to maintain a constant force in deafferented patients - they were able to maintain a constant force with visual feedback, but the force output drifted shortly after visual feedback was removed (see Fig. 15 from Rothwell et al. 1982). The variability in the force output may come from multiple sources, including variability in the centrally generated motor command and motor noise (Jones et al. 2002). This suggests that afferent feedback is necessary to recalibrate force output. However, the role of specific afferents is unclear because inputs from cutaneous, joint and muscle afferents are all removed in these patients.

Here, we disrupted cutaneous afferents using peripheral nerve stimulation. The advantage of using electrical nerve stimulation is that we could quickly switch between normal and altered cutaneous feedback conditions, which could not be done using anesthesia. Ankle dorsiflexion force errors increased after sudden disruption of cutaneous feedback in the foot dorsum via peroneal nerve stimulation, suggesting that subjects were unable to rely on central signals (e.g., efferent copy) and other sensory feedback (e.g., changes in muscle length and tension) to recalibrate the force output in this situation.

Another possibility is that stimulation increased the amplitude of the cutaneous afferent signal, which the nervous system would interpret as an increase in pressure (i.e., pushing harder). Thereby, subjects should under-shoot when the dorsum is stimulated and over-shoot when the sole is stimulated. We think this is unlikely because the mean force output did not show consistent changes across trials when the simulation was applied.

Providing visual feedback during repetitive electrical nerve stimulation improved the ability to maintain force output accurately. During continuous peroneal nerve stimulation, subjects were able to maintain the target force after visual feedback was removed. This suggests that the nervous system may rely on other available inputs (e.g., central commands, muscle spindle) to recalibrate force output under the circumstances.

The phenomenon of sensory weighting has been demonstrated in the estimation of position during reaching; the nervous system can dynamically weight and combine multiple sources when sufficient information about their accuracy is known (Beers et al. 1996; Ernst and Banks 2002). The nervous system appears to optimize the weights of different sensory modalities (e.g., vision, proprioception) in order to minimize the variance of the final estimate of position (Ernst and Banks 2002). Here, we showed that the nervous system might also vary the way it integrates efferent and afferent signals to form estimates of force output.

\section{Position control}

We found no effects of repetitive electrical nerve stimulation on the perception of ankle joint position. This does not imply that cutaneous input has no contribution to the sense of position. The effects of peroneal nerve and plantar nerve stimulation were felt on the skin of the foot, and therefore, we did not expect a profound effect on position sense at the ankle joint. Other studies have suggested that cutaneous input likely contributes to position sense because anesthesia impairs movement detection in the thumb (Gandevia and McCloskey 1976) and ankle joint (Lowrey et al. 2010). Moreover, microneurographic studies suggest that cutaneous afferents can potentially encode position information about hand (Edin and Abbs 1991), knee (Edin 2001) and ankle (Aimonetti et al. 2007) movements. Cutaneous input may also have a role in biasing position sense at extreme joint positions where the skin is most stretched (Fuentes and Bastian 2010).

\section{Functional relevance}

Ankle dorsiflexion is an important function during walking, allowing the toes to clear the ground during the swing phase of walking. Insufficient activation of the tibialis anterior muscle in persons post-stroke and children with cerebral palsy is related to particular gait deficits (e.g., foot drop). One training approach using a resistive ankle-foot 
orthosis has been proposed that could promote increased activation in ankle dorsiflexion muscles during walking (Blanchette et al. 2011). The training paradigm is based on trial-and-error adaptation that acts to account for predictable perturbations (Lam et al. 2006; Choi and Bastian 2007; Blanchette and Bouyer 2009). We hypothesize that unexpected contact force on the foot dorsum may be an important error signal driving motor adaptation, and we are currently investigating whether disruption of cutaneous feedback via stimulation of the foot dorsum or foot sole alters adaptation to ankle torque perturbations during walking. If cutaneous feedback contributes to ankle torque correction, it may be possible to augment the training effects using cutaneous feedback.

\section{Conclusion}

In conclusion, we have shown that the nervous system uses cutaneous feedback in combination with other inputs to estimate force about the ankle control. In this study, we showed that electrical stimulation of peripheral nerves disrupted cutaneous afferent information and decreased accuracy in ankle force control. Future studies will be necessary to further understand how somatosensory signals influence motor learning. Understanding how the nervous system combine and use various inputs will help us identify which functional aspects are impaired in aging and neurological diseases and how to assist these populations.

Acknowledgments Support for this work was provided by the Danish Research Council (11-107721/FSS) and by the Whitaker International Scholars Program.

\section{References}

Aimonetti J-M, Hospod V, Roll J-P, Ribot-Ciscar E (2007) Cutaneous afferents provide a neuronal population vector that encodes the orientation of human ankle movements. J Physiol (Lond) 580:649-658

Arezzo I, Schaumburg H, Laudadio C (1985) The vibratron: a simple device for quantitative evaluation of tactile/vibratory sense. Neurology 35:169

Beers R, Sittig A, Denier J (1996) How humans combine simultaneous proprioceptive and visual position information. Exp Brain Res 111:253-261

Blanchette A, Bouyer LJ (2009) Timing-specific transfer of adapted muscle activity after walking in an elastic force field. J Neurophysiol 102:568-577

Blanchette A, Lambert S, Richards CL, Bouyer LJ (2011) Walking while resisting a perturbation: effects on ankle dorsiflexor activation during swing and potential for rehabilitation. Gait Posture 34(3):358-363

Choi JT, Bastian AJ (2007) Adaptation reveals independent control networks for human walking. Nat Neurosci 10:1055-1062

Edin BB (2001) Cutaneous afferents provide information about knee joint movements in humans. J Physiol (Lond) 531:289-297
Edin BB, Abbs JH (1991) Finger movement responses of cutaneous mechanoreceptors in the dorsal skin of the human hand. J Neurophysiol 65:657-670

Ernst MO, Banks MS (2002) Humans integrate visual and haptic information in a statistically optimal fashion. Nature 415:429-433

Fuentes CT, Bastian AJ (2010) Where is your arm? Variations in proprioception across space and tasks. J Neurophysiol 103:164-171

Gandevia SC, Mccloskey DI (1976) Joint sense, muscle sense, and their combination as position sense, measured at distal interphalangeal joint of middle finger. J Physiol (Lond) 260:387-406

Gandevia SC, Mccloskey DI (1977a) Changes in motor commands, as shown by changes in perceived heaviness, during partial curarization and peripheral anaesthesia in man. J Physiol 272:673-689

Gandevia SC, Mccloskey DI (1977b) Effects of related sensory inputs on motor performances in man studied through changes in perceived heaviness. J Physiol 272:653-672

Gandevia SC, Macefield VG, Bigland-Ritchie B, Gorman RB, Burke D (1993) Motoneuronal output and gradation of effort in attempts to contract acutely paralysed leg muscles in man. J Physiol 471:411-427

Henningsen H, Ende-Henningsen B, Gordon A (1995) Contribution of tactile afferent information to the control of isometric finger forces. Exp Brain Res 105:312-317

Henningsen H, Knecht S, Endehenningsen B (1997) Influence of afferent feedback on isometric fine force resolution in humans. Exp Brain Res 113:207-213

Jones LA, Hunter IW (1983) Effect of fatigue on force sensation. Exp Neurol 81:640-650

Jones K, Hamilton A, Wolpert D (2002) Sources of signal-dependent noise during isometric force production. J Neurophysiol 88:1533-1544

Lam T, Anderschitz M, Dietz V (2006) Contribution of feedback and feedforward strategies to locomotor adaptations. J Neurophysiol 95:766-773

Lowrey CR, Strzalkowski NDJ, Bent LR (2010) Skin sensory information from the dorsum of the foot and ankle is necessary for kinesthesia at the ankle joint. Neurosci Lett 485:6-10

Macefield G, Gandevia SC, Burke D (1990) Perceptual responses to microstimulation of single afferents innervating joints, muscles and skin of the human hand. J Physiol (Lond) 429:113-129

Marsden CD, Rothwell JC, Traub MM (1979) Effect of thumb anaesthesia on weight perception, muscle activity and the stretch reflex in man. J Physiol 294:303-315

Maschke M, Tuite PJ, Krawczewski K, Pickett K, Konczak J (2006) Perception of heaviness in parkinson's disease. Mov Disord 21:1013-1018

Nasseri K, Strijers RL, Dekhuijzen LS, Buster M, Bertelsmann FW (1998) Reproducibility of different methods for diagnosing and monitoring diabetic neuropathy. Electromyogr Clin Neurophysiol 38:295-299

Ochoa J, Torebjörk E (1983) Sensations evoked by intraneural microstimulation of single mechanoreceptor units innervating the human hand. J Physiol 342:633-654

Rothwell JC, Traub MM, Day BL, Obeso JA, Thomas PK, Marsden CD (1982) Manual motor performance in a deafferented man. Brain 105:515-542

Sanes JN, Mauritz KH, Evarts EV, Dalakas MC, Chu A (1984) Motor deficits in patients with large-fiber sensory neuropathy. Proc Natl Acad Sci 81:979-982

Simon AM, Kelly BM, Ferris DP (2009) Sense of effort determines lower limb force production during dynamic movement in individuals with poststroke hemiparesis. Neurorehabil Neural Repair 23:811-818

Vallbo ÅB, Olsson KÅ, Westberg K-G, Clark FJ (1984) Microstimulation of single tactile afferents from the human hand. Brain 107:727-749 